\title{
Study on risk assessment of expressway nighttime maintenance construction: A Dynamic Bayesian Network model
}

\author{
Zhen Tian ${ }^{1, *}$, Jinhua Fan ${ }^{1}$, Qianqian $\mathrm{Chen}^{1}$, Huaichen $\mathrm{Hu}^{1}$, Yanyang Shen ${ }^{1}$ \\ ${ }^{1}$ South China University of Technology, School of Mechanical and Automotive Engineering, 510641Wushan Road, Guangzhou, \\ Guangdong, PR China
}

\begin{abstract}
There are many risk factors and large uncertainties in expressway nighttime maintenance construction(ENMC), and the state of risk factors will change dynamically with time. In this study, a Dynamic Bayesian Network (DBN) model was proposed to investigate the dynamic characteristics of the timevarying probability of traffic accidents during expressway maintenance at night. Combined with Leaky Noisyor gate extended model, the calculation method of conditional probability is determined. By setting evidences for DBN reasoning, the time series change curve of the probability of traffic accidents and other risk factors are obtained. The results show that DBN can be applied to risk assessment of ENMC.
\end{abstract}

\section{Introduction}

Accident statistical analysis and research showed that the accident rate and fatality rate of nighttime maintenance construction operations are much higher than that of day maintenance construction[1]. In order to analyze the risk of expressway construction work area, Kairan Zhang et al. [2], Yingfeng Li et al.[3], Xianghai Meng et al.[4], Sze and Soong[5], and Higa et al.[6] identified operationrelated risk factors. Xinxin Wei et al.[7] analyzed accidents under different illumination conditions. The severity was studied. Biao $\mathrm{Wu}$ et al.[8] conducted risk assessment on risk factors in the work area; Jikun Liu et al.[9]proposed a LECT evaluation method that can identify key risk factors in the operation process; Rahman et al.[10] studied the effect of dynamic information signs on controlling the speed of the driver. The current researchers mainly focus on the identification and evaluation of risk factors in the daytime maintenance of expressways or the construction operations of new construction, renovation and expansion. However, ENMC's quantitative risk analysis is still lack of systematic research. DBN is a higher efficient approach to risk analysis[11]. Therefore, it is a very important way to quantitatively analyze the risks of ENMC through the DBN method.

\section{Dynamic Bayesian Network}

A DBN is a Bayesian network extended with additional mechanisms that are capable of modeling influences over time [12]. Hidden Markov model is considered the simplest DBN[13]. Due to the system change characteristics of the DBN, directly perform analysis and modeling is hard to obtain. Therefore, in order to minimize the difficulty of usage, the two hypotheses are proposed as follow:

(1) Markov hypothesis: the future state is only related to the present and has nothing to do with the past.

(2) Static hypothesis: adjacent random processes within a finite time are stable and consistent at any time $t$.

The general structure of the DBN is shown in Fig.1.

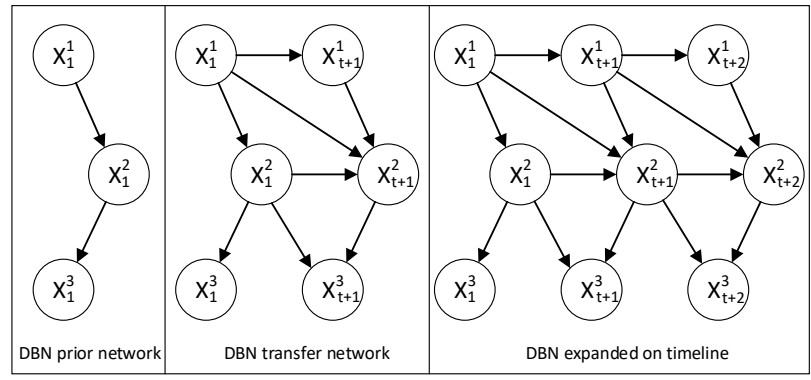

Fig.1 The general structural of the DBN

\section{Methodology}

\subsection{Defining the risk factors used in the ENMC model}

According to literature review, our study is to investigate the ENMC workplace, including conduct lots of surveys with relevant personnel. The Operational risk factors were identified through a combination of literature review, onsite investigation and personnel interviews. The risk factors are divided into 5 categories: Driver(D), environment(E), Highway(H), Vehicle(V), and Workzone $(\mathrm{W})$, and corresponding secondary indicators are shown in Table 1.

\footnotetext{
* Corresponding author: zhentian@scut.edu.cn
} 
Table 1. Description of risk factor

\begin{tabular}{|c|c|c|c|}
\hline \multicolumn{3}{|c|}{ No. Risk factor } & Description \\
\hline 1 & $\mathrm{D}$ & D1 & Driving experience \\
\hline 2 & & D2 & Night line of sight \\
\hline 3 & & D3 & over-speed driving \\
\hline 4 & & D4 & Fatigue driving \\
\hline 5 & & D5 & Dangerous driving \\
\hline 6 & & D6 & Aggressive driving \\
\hline 7 & & D7 & Driving error \\
\hline 8 & E & E1 & temperature \\
\hline 9 & & E2 & Light \\
\hline 10 & & E3 & weather \\
\hline 11 & & E4 & Traffic volume \\
\hline 12 & & E5 & Traffic composition \\
\hline 13 & & E6 & Indicator visibility \\
\hline 14 & $\mathrm{H}$ & H1 & Speed limit \\
\hline 15 & & $\mathrm{H} 2$ & Number of lanes \\
\hline 16 & & $\mathrm{H} 3$ & Road conditions \\
\hline 17 & & $\mathrm{H} 4$ & Geometric Features \\
\hline 18 & & $\mathrm{H} 5$ & Maintenance location \\
\hline 19 & $\mathrm{~W}$ & W1 & Operator fatigue \\
\hline 20 & & W2 & Unsafe behavior of workers \\
\hline 21 & & W3 & Job skills and knowledge mastery level \\
\hline 22 & & W4 & $\begin{array}{l}\text { Pre-job education and training and } \\
\text { clarification }\end{array}$ \\
\hline 23 & & W5 & Illegal command \\
\hline 24 & & W6 & Operation error \\
\hline 25 & & W7 & Guardianship error \\
\hline 26 & & W8 & Work area settings \\
\hline 27 & & W9 & Work area lighting \\
\hline 28 & & W10 & Traffic safety facilities \\
\hline 29 & & W11 & Construction time \\
\hline 30 & $\mathrm{~V}$ & V1 & Vehicle Type \\
\hline 31 & & $\mathrm{~V} 2$ & Overload or overrun \\
\hline 32 & & V3 & Brake failure of vehicle \\
\hline 33 & & V4 & Tire burst or drop \\
\hline 34 & & V5 & The vehicle is out of control \\
\hline 35 & & V6 & Construction vehicle breakdown \\
\hline 36 & & V7 & $\begin{array}{l}\text { Construction vehicles enter and exit the } \\
\text { work area }\end{array}$ \\
\hline
\end{tabular}

\subsection{Defining the structure of the ENMC model}

As a modeling tool dedicated to the construction and analysis of graphical decision-making theory models [14], the professional analysis software GeNIe3.0 (academic version) can be used to build a probability assessment models and data inference.

Input the basic data into the Bayesian network constructed by the GeNIe, performing DBN inference, and selecting the prior probability of key time nodes for real-time updating. The dynamic reasoning results are revised and integrated through machine learning to realize the mutual complementation of mathematical theories and expert knowledge, thereby further improving the scientificity and rationality of the evaluation results.

According to the risk events and secondary indicators identified in Table 1, the DBN model shown in Figure 2 is constructed. Where $\mathrm{R}$ represents the risk of traffic accidents in the workplace.

During the site investigation, we found that the time of ENMC is a total of 9 hours usually from 9:00 PM to 6:00 AM the next day, so the DBN constructed in this study can establish an hourly time point to the event node, A total of 10 time steps to represent the changing events evolution mechanism (The temporal plate division can be adjusted according to the actual situation of each place). The proposed model can be seemed as a tool to calculate the traffic accident risk of ENMC. The time dependence of nodes and arcs in the model can be adjusted according to the conditions of a specific area. This model is designed to support time-related operational decision-making to reduce the value of the node $\mathrm{R}$ (risk of traffic accidents in the work area), such as adjusting traffic volume and selecting vehicle speed limits. For example, in this study, we assumed that ENMC is in progressing, and the timeseries changes of traffic volume and operation area in the area are constantly observed during the operation. The correlation among those indicators can be reflected by pointed arrows in the GENIE3.0. The directed arc segment "1" on each parent node indicates that the time interval between adjacent time slices is 1 hour. We can clearly displays those indicators and its sub-indicators by different colours as shows in Fig.2.

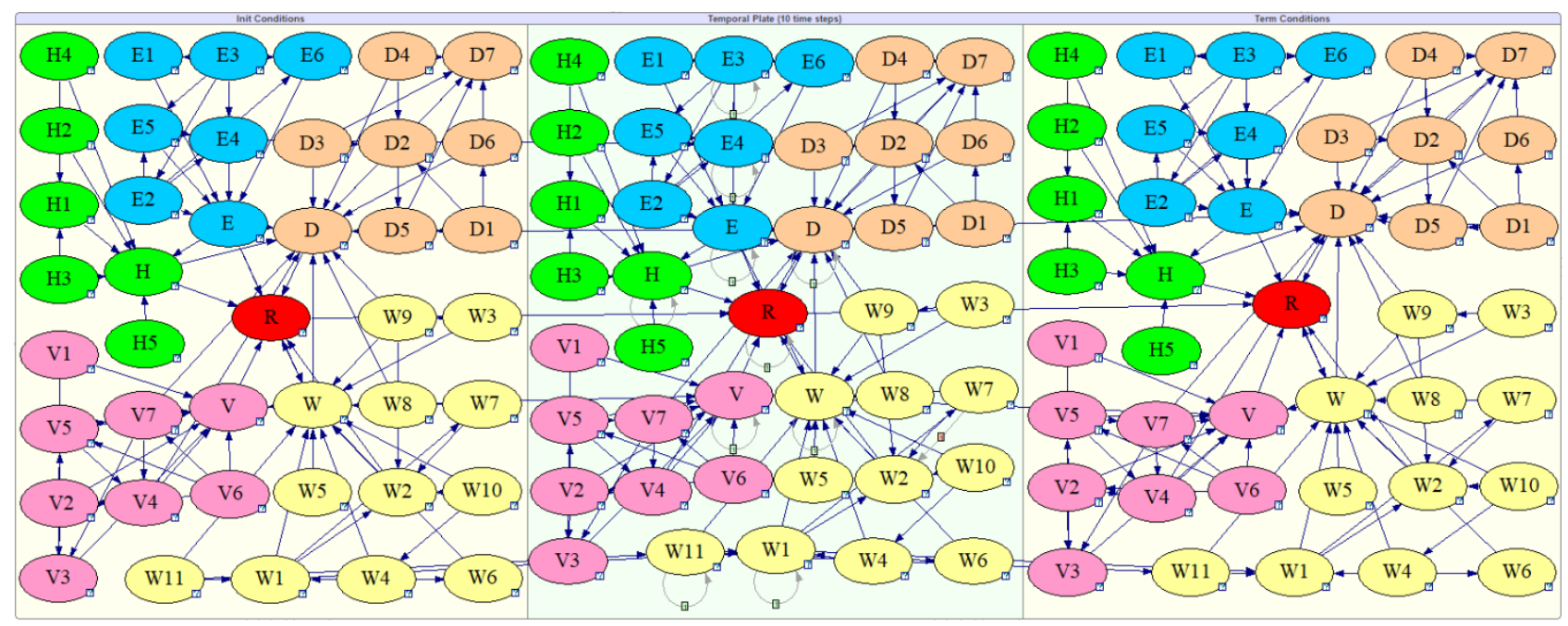

Fig. 2 DBN model structure of ENMC 


\subsection{Conditional probability table}

By experts scoring to the probability of each time state, the prior probability, conditional probability and original transition probability of all the parent nodes of an event are obtained. To standardize the probabilistic expression during scoring and make the scoring data easy to refer to and distinguish, the 7-level risk probability expression method proposed from the United Nations Intergovernmental Panel on Climate Change (IPCC) is adopted to our study[16]. Considering the statistical data of expressway traffic accidents studied from a China expressway management company, the probabilities of traffic accidents are ranking in $10^{-5}$ to $10^{-4}$. In the study, all of values of those probabilities are multiplied by $10^{-4}$ for sake of take the risk analysis into the most dangerous place. In next stage, based on the relevant data by the expert survey method, the conditional probability table of the entire network is calculated according to the noisy-orgate model[17]. The prior probability, conditional probability and original transition probability are input to GENIE3.0 for DBN inference. Because of space limits of this paper, only node E5 can be regarded as an example to present the conditional probability table, as shown in Fig.3. Y represents the occurrence or development of the risk factor in the wrong direction, while $\mathrm{N}$ is the opposite of $\mathrm{Y}$.

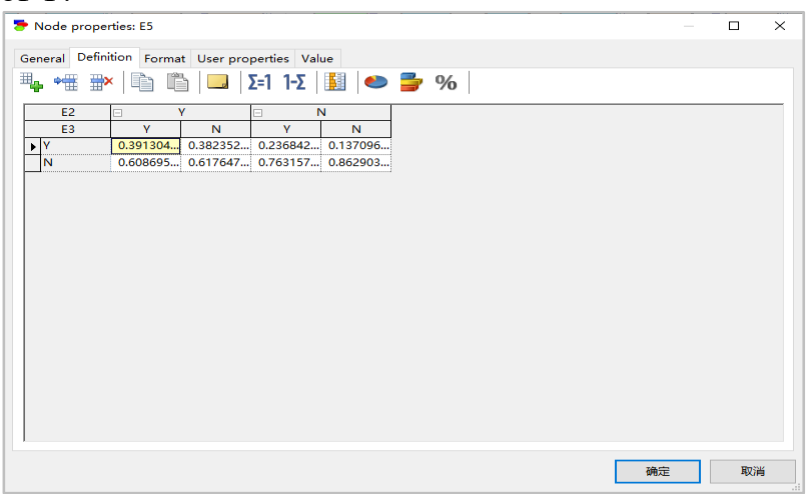

Fig. 3 Conditional probability table for the variable E5

\section{Scenario analysis and evaluate of DBN model}

In this study, four nodes E4, D, W1 and V2 are used for evidence setting. Fig. 4 shows the evidence settings for the four nodes. These evidences are not set randomly, which should be according to the actual characteristics of the expressway at night. For example, in the case of E4 (traffic volume), when the traffic is heavy from 9:00 PM to 12:00 PM, we can select "Y" to indicate the occurrence of risk event. From 0:00 AM to 5:00AM, we can choose "N" when the traffic volume decreases. When the traffic volume increases after 5:00 AM, we can choose "Y". The other three "Evidence" settings also follow the above logic, so these four evidences can be established.

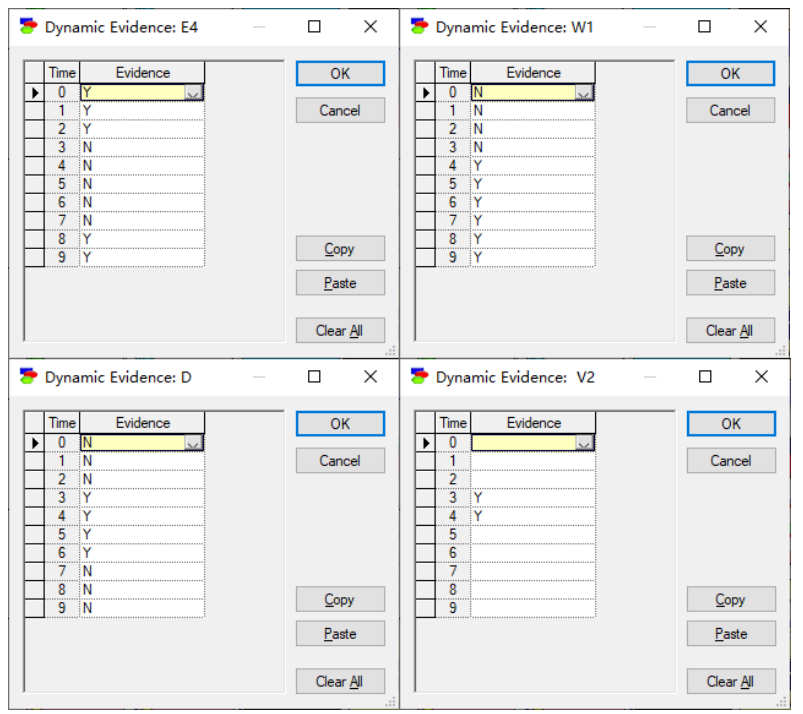

Fig. 4 Evidence setting

Input the evidence into the DBN model to perform DBN forward inference, and the results are shown in Fig.5, Fig. 6 and Table 2. Among them, $\mathrm{R}$ in Table 2 and Fig. 5 represents the probability of a traffic accident in a hazard state at ENMC.

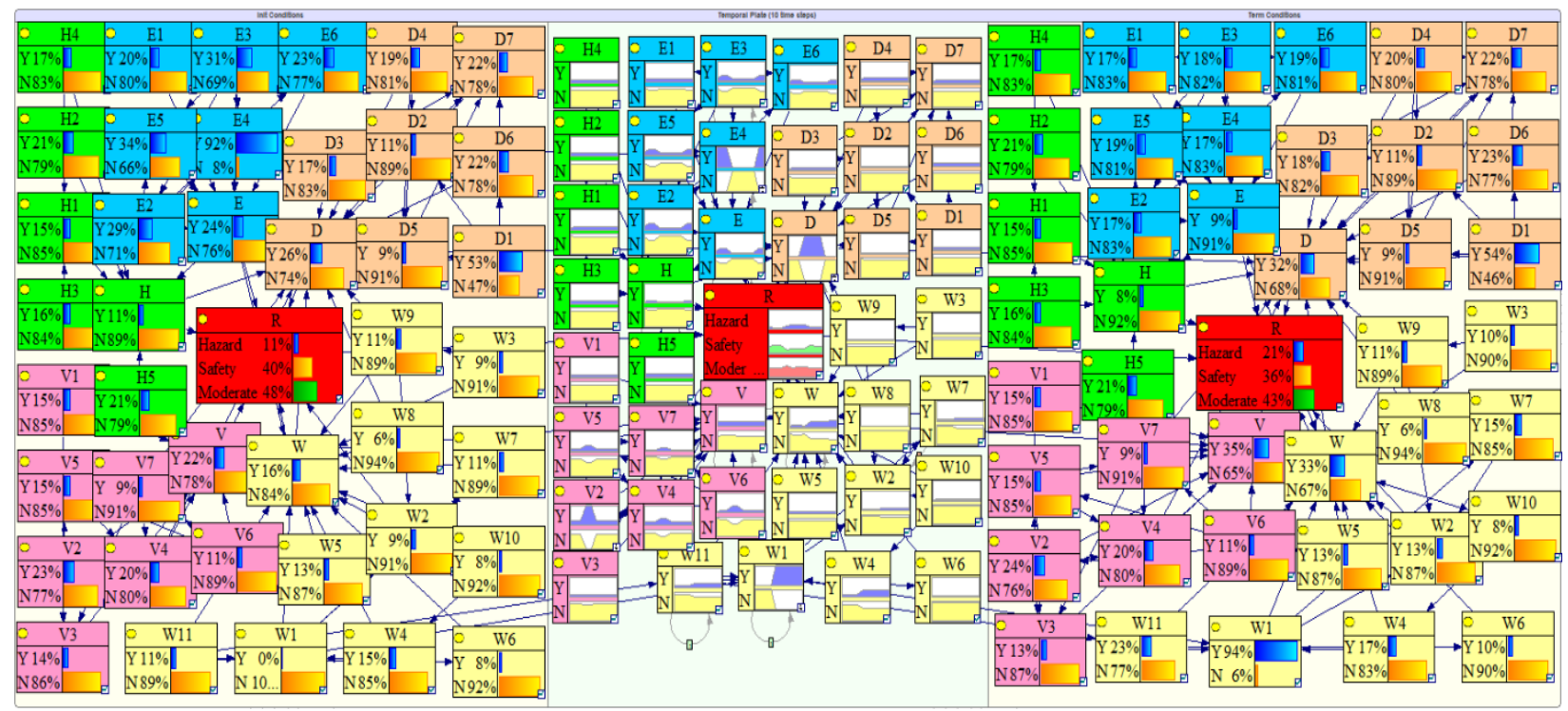

Fig. 5 DBN model with evidence input 
Table 2. Dynamic reasoning results

\begin{tabular}{|c|c|c|c|c|c|c|c|c|c|c|}
\hline Node & $\mathrm{T}=0$ & $\mathrm{~T}=1$ & $\mathrm{~T}=2$ & $\mathrm{~T}=3$ & $\mathrm{~T}=4$ & $\mathrm{~T}=5$ & $5 \mathrm{~T}=6$ & $5 \mathrm{~T}=7$ & $\mathrm{~T}=8$ & $\mathrm{~T}=9$ \\
\hline E1 & 0.17 & 0.20 & 0.20 & 0.17 & 0.17 & 0.17 & 70.17 & 70.17 & 0.20 & 0.20 \\
\hline 2 & .18 & & 0.31 & 0.14 & 0.14 & 0.14 & 40.14 & 0.14 & 0.31 & 0.31 \\
\hline 3 & 0.19 & 0.33 & 0.33 & 0.15 & 0.15 & 0.15 & 50.15 & 0.15 & 0.33 & 0.33 \\
\hline 4 & 1 & 1 & 1 & 0 & 0 & 0 & 0 & 0 & 1 & 1 \\
\hline & 0.21 & 0.36 & 0.36 & 0.15 & 0.15 & 0.15 & 50.15 & 50.15 & 0.36 & 0.36 \\
\hline 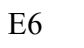 & 0.19 & & 0.24 & 0.18 & 0.18 & 0.18 & 80.18 & 0.18 & 0.24 & 0.24 \\
\hline & 0.16 & & 0.27 & 0.06 & 0.06 & 0.06 & 60.06 & 50.06 & 0.27 & 0.27 \\
\hline & 0.15 & & 0.15 & 0.15 & 0.15 & 0.15 & 50.15 & 50.15 & 0.15 & 0.15 \\
\hline 72 & 0.21 & 0.21 & 0.21 & 0.21 & 0.21 & 0.21 & 10.21 & 0.21 & 0.21 & 0.21 \\
\hline H3 & 0.16 & 0. & 0.16 & 0.16 & 0.16 & 0.16 & 60.16 & 50.16 & 0.16 & 0.16 \\
\hline $\mathrm{H} 4$ & 0.17 & 0.17 & 0.17 & 0.17 & 0.1 & 0.17 & 70.17 & 70.17 & 0.17 & 0.17 \\
\hline H5 & 0.21 & 0.21 & 0.21 & 0.21 & 0.2 & 0.21 & 10.21 & 0.21 & 0.21 & 0.21 \\
\hline $\mathrm{H}$ & 0.09 & 0. & 0. & 0.08 & 0. & 0.08 & 80.08 & 0.08 & 0.11 & 0.11 \\
\hline eD1 & 0.16 & & 0.17 & 0.17 & 0.17 & 0.17 & 70.17 & 70.17 & 0.17 & 0.17 \\
\hline ? & & & & & & 0.10 & & & & 0.10 \\
\hline & & & & & & & & & & 0.18 \\
\hline דינ & & & & & & 0.20 & & & & 0.20 \\
\hline 55 & 0.06 & & 0.0 & 0.08 & & 0.08 & $\begin{array}{ll}80.08 \\
\end{array}$ & 0.08 & 0.08 & 0.08 \\
\hline 66 & 0.15 & & 0.17 & 0.17 & 0.17 & 0.17 & 70.17 & 70.17 & 0.17 & 0.17 \\
\hline D7 & 0.17 & 0.21 & 0.21 & 0.21 & 0.21 & 0.21 & 10.21 & 0.21 & 0.21 & 0.21 \\
\hline & 0 & 0 & 0 & 1 & 1 & 1 & 1 & 0 & 0 & 0 \\
\hline $\mathrm{V} 1$ & 0.15 & 0.15 & 0.15 & 0.15 & 0.15 & 0.15 & 50.15 & 50.15 & 0.15 & 0.15 \\
\hline $\mathrm{V} 2$ & 0.23 & 0.24 & 0.24 & 1 & 1 & 0.24 & 40.24 & 0.24 & 0.24 & 0.24 \\
\hline $\mathrm{V} 3$ & 0.13 & 0.13 & 0.13 & 0.18 & 0.18 & 0.13 & 30.13 & 0.13 & 0.13 & 0.13 \\
\hline V4 & 0.19 & 0.20 & 0.20 & 0.36 & 0.36 & 0.20 & 00.20 & 0.20 & 0.20 & 0.20 \\
\hline V5 & 0.15 & 0.15 & 0.15 & 0.32 & 0.32 & 0.15 & 50.15 & 50.15 & 0.15 & 0.15 \\
\hline V6 & 0.11 & 0.11 & 0.11 & 0.33 & 0.33 & 0.11 & 10.11 & 0.11 & 0.11 & 0.11 \\
\hline V7 & .08 & 0.09 & 0.09 & 0.21 & & 0.09 & 90.09 & 0.09 & 0.09 & 0.09 \\
\hline V & 0.27 & 0.22 & 0.22 & 0.24 & 0.29 & 0.28 & $\begin{array}{ll}8 & 0.28\end{array}$ & 0.28 & 0.29 & 0.29 \\
\hline W1 & 0 & 0 & 0 & 0 & 1 & 1 & 1 & 1 & 1 & 1 \\
\hline W2 & 0.08 & 0.09 & 0.09 & 0.09 & 0.14 & 0.14 & 40.14 & 0.14 & 0.1 & 0.14 \\
\hline W3 & 0.09 & 0.10 & 0.10 & 0.10 & 0.10 & 0.10 & $\begin{array}{ll}0 & 0.10\end{array}$ & 0.10 & 0.10 & 0.10 \\
\hline W4 & 0.16 & 015 & 0.15 & 0.15 & 0.36 & 0.36 & 60.36 & 50.36 & 0.36 & 0.36 \\
\hline W5 & 0.12 & 013 & 0.13 & 0.13 & 0.13 & 0.13 & 30.13 & 0.13 & 0.13 & 0.13 \\
\hline W6 & 0.08 & 008 & 0.08 & 0.08 & 0.10 & 0.10 & 00.10 & 0.10 & 0.10 & 0.10 \\
\hline W7 & 0.10 & 011 & 0.11 & 0.11 & 0.15 & 0.15 & 50.15 & 50.15 & 0.15 & 0.15 \\
\hline W8 & 0.05 & & & & & 0.06 & 60.06 & 50.06 & 0.06 & 0.06 \\
\hline W9 & 0.10 & 0.11 & 0.11 & 0.11 & & 0.11 & 10.11 & 0.11 & 0.11 & 0.11 \\
\hline W10 & 0.07 & 0.08 & 0.08 & 0.08 & 0.10 & 0.10 & 00.10 & 0.10 & 0.10 & 0.10 \\
\hline W11 & 0.11 & 0.12 & 0.12 & 0.12 & 0.23 & 0.23 & $\begin{array}{ll}30.23 \\
\end{array}$ & 0.23 & 0.23 & 0.23 \\
\hline W & & & & & 0. & 0. & 40. & 0.34 & 0.38 & 0.38 \\
\hline & 0.13 & 0.08 & 0.08 & 0.21 & 0.24 & 0.24 & 40.24 & 0.12 & 0.14 & 0.14 \\
\hline
\end{tabular}

We can see from Fig.5 that the probability of risk factor D1 is the only node exceeding 50\%, Node E4 and $\mathrm{W} 1$ are affected by the evidence input, and they are actually $7 \%$ and $17 \%$ respectively when no evidence is input, indicating that the node D1 is the most significant risk in the driver dimension factor with $54 \%$. The most noteworthy of the other four dimensions are $\mathrm{H} 2$ (21\%) , V2 (24\%), E5 (15\%) and W4 (17\%).
Among the five first-level indicators, except for the decline in the probability of nodes $\mathrm{H}$ and $\mathrm{E}$, the other three have a significant increase. The changing trends of all the first-level indicators are $\mathrm{H}(-3 \%), \mathrm{D}(+8 \%), \mathrm{V}(+13 \%), \mathrm{W}$ $(14 \%)$ and $\mathrm{E}(-7 \%)$. The risk of $\mathrm{R}$ in the hazard state has risen from $11 \%$ to $21 \%$. The other remaining nodes' probability has changed not over $5 \%$.
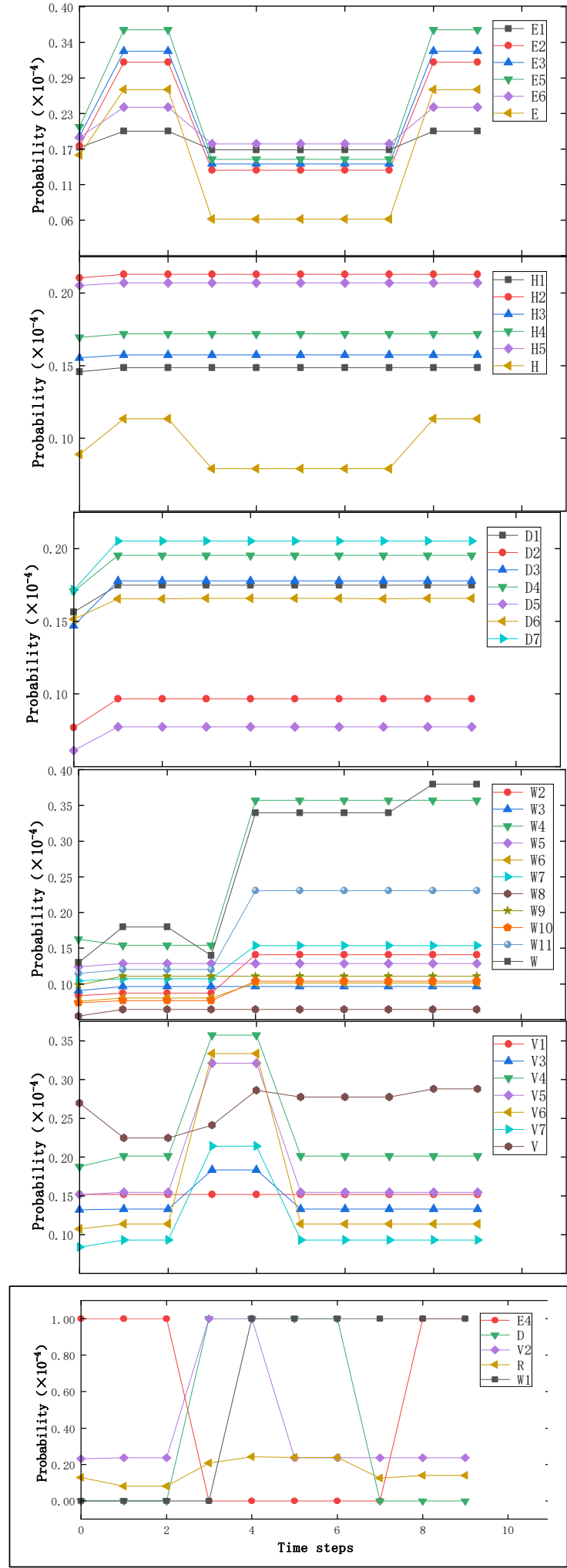

Fig. 6 Time steps variable curves of probability of risk factors 
It can be seen from Table 2 and Figure 5 that after setting the evidence node, the secondary indicators in the $\mathrm{E}$ dimension are obviously affected by E4, and the trend is opposite to E4. The secondary indicators in the H and D dimensions are less affected, and the probability of occurrence only increases slightly when the time steps are $0 \sim 1$. The change trend of the second-level indicator probability in the $\mathrm{W}$ dimension is consistent with the $\mathrm{W} 1$ evidence setting, indicating that $\mathrm{W} 1$ is positively correlated with other second-level indicators. The change trend of $\mathrm{R}$ present a $\mathrm{W}$ shape, and the probability has gone through two processes of first falling and then rising, demonstrating that the four evidence nodes have a significant impact on $\mathrm{R}$.

\section{Conclusions}

The risk factors of EMNC operations are uncertain and are prone to dynamic changes over time. In this paper, we had proposed a DBN to investigate the dynamic characteristics of the time-varying probability of traffic accidents during expressway maintenance at night. The results show that node $\mathrm{D} 1$ (driver skill) is the most significant factor for node $\mathrm{R}$ (traffic accident). The DBN is capable to simulate temporal relationships in ENMC. Besides, a Chinese related company has also participated in the data collecting and provided historic statistical probability in traffic accidents. Combined with Leaky Noisy-or gate extended model, the calculation method of conditional probability is determined. By setting evidences for DBN reasoning, the time series change curve of the probability of traffic accidents and other risk factors are obtained.

In the case of setting evidence, risk can also be quantified. These quantitative risk assessments provide a basis to dynamic risk management and control on the workplace along with improve the accuracy of risk estimation. The results show that DBN can be applied to risk assessment of ENMC and other critical process construction work systems.

\section{References}

1. Cottrell Jr, Benjamin H. "Improving night work zone traffic control." [R].Virginia Transportation Research Council, (1999).

2. Zhang, K., and M. Hassan. "Crash severity analysis of nighttime and daytime highway work zone crashes." PLOS ONE 14(2019).

3. Li Y, and Yong B. "Highway work zone risk factors and their impact on crash severity." Journal of Transportation engineering 135.10 (2009): 694-701. (in Chinese)

4. Meng X, Xu H, Shi Y. " Identification of Rear-end Collision Risk on Freeway Work Zone and Its Prominent Influencing Factors." Journal of Highway and Transportation Research and Development 29.12 (2013): 133-138. (in Chinese)

5. Sze, N. N., and Ziqi Song. "Factors contributing to injury severity in work zone related crashes in New
Zealand." International journal of sustainable transportation 13.2 (2019): 148-154.

6. Higa, Lance, and Jin-Lee Kim. "Evaluating accident data for the safety of nighttime construction in Southern California." ICSDEC 2012: Developing the Frontier of Sustainable Design, Engineering, and Construction. (2013): 711-718.

7. Wei X, Shu X, Huang B, et al. Analyzing traffic crash severity in work zones under different light conditions $[\mathrm{J}]$. Journal of advanced transportation, 2017.

8. Wu B, Xu H, and Dai T. " Identifying Safety Factors on Highway Work Zone Based on DEMATEL and ISM." Journal of Transportation Systems Engineering and Information Technology $\mathbf{1 0 . 5}$ (2010): 130-136. (in Chinese)

9. Liu J, Liu E, Guo H. " Research on Risk Evaluation Model of Construction Processes in Mountain Area Highways." Journal of China \& Foreign Highway 39.03(2019):313-317. (in Chinese)

10. Rahman M M, Strawderman L, Garrison T, et al. Work zone sign design for increased driver compliance and worker safety[J]. Accident Analysis \& Prevention 106 (2017): 67-75.

11. Anitha G, Priya S B. Posture based health monitoring and unusual behavior recognition system for elderly using dynamic Bayesian network[J]. Cluster Computing, 22.6(2019): 13583-13590.

12. Sarshar, P., J. Radianti, and J. J. Gonzalez. "Modeling panic in ship fire evacuation using dynamic Bayesian network." Third International Conference on Innovative Computing Technology IEEE, 2013.

13. Khan, Bushra, Faisal Khan, and Brian Veitch. "A Dynamic Bayesian Network model for ship-ice collision risk in the Arctic waters." Safety Science 130 (2020): 104858

14. BayesFusion, GeNIe. Retrieved from http://www.ba yesfusion.com, 2021.

15. Oniśko, A. "Application of dynamic Bayesian netwo rks to risk assessmnet in medicine." Zeszyty Nauko we Politechniki Białostockiej. Informatyka 5(2010): 35-49.

16. Patt, A. G., and D. P. Schrag. "Using Specific Langu age to Describe Risk and Probability." Climatic Cha nge 61.1(2003): 17-30.

17. Liao, F., Liang, M., Li, Z., Hu, X., Song, S.. "Evalua te the malignancy of pulmonary nodules using the 3$\mathrm{d}$ deep leaky noisy-or network." IEEE transactions o $\mathrm{n}$ neural networks and learning systems 30.11 (2019): 3484-3495. 